\title{
Availability and affordability of medicines and diagnostic tests recommended for management of asthma and chronic obstructive pulmonary disease in sub-Saharan Africa: a systematic review
}

Davis Kibirige ${ }^{1,2^{*}}$, Richard E. Sanya ${ }^{2}$, Rebecca Nantanda ${ }^{3,4}$, William Worodria ${ }^{5}$ and Bruce Kirenga $a^{3,5}$

\begin{abstract}
Background: Early accurate diagnosis and sustainable availability of affordable medicines and diagnostic tests is fundamental in optimal management of asthma and chronic obstructive pulmonary disease (COPD). We systematically reviewed original research articles about availability and affordability of medicines and diagnostic tests recommended for management of asthma and COPD in sub-Saharan Africa (SSA).

Methods: We searched PubMed, Scopus and African Journal Online for original research articles conducted in SSA between 2000 and March 2018 containing information about availability and affordability of any recommended medicine and diagnostic test for asthma and COPD.

Results: The search yielded 9 eligible research articles. Availability of short-acting beta agonists (SABA), inhaled corticosteroids (ICS) and short acting anti-muscarinic agents (SAMA) ranged between 19.9-100\%, 0-45.5\% and $0-14.3 \%$ respectively. Combination of ICS-long acting beta agonists (LABA) were available in $0-14.3 \%$ of facilities surveyed. There was absence of inhaled long acting anti-muscarinic agents (LAMA) and LAMA/LABA combinations. Spirometry and peak expiratory flow devices were available in $24.4-29.4 \%$ and $6.7-53.6 \%$ respectively. Affordability of SABA and ICS varied greatly, ranging from $<2$ to 107 days' wages while ICS-LABA combinations, SAMA and oral theophylline plus leukotriene receptor antagonists cost 6.4-17.1, 13.7 and 6.9 days' wages respectively.

Conclusion: Availability and affordability of medicines and diagnostics recommended for the management of asthma and COPD is a big challenge in SSA. Research about this subject in this region is still limited. More robustly performed studies are required to further understand the magnitude of inequity in access to these medicines and diagnostic tests in SSA and also to formulate simple pragmatic solutions to address this challenge.
\end{abstract}

Keywords: Availability, Affordability, Essential medicines, Diagnostic tests, Asthma, Chronic obstructive pulmonary disorders, COPD, Sub-Saharan Africa, Africa

\footnotetext{
*Correspondence: kibirigedavis@gmail.com

${ }^{1}$ Department of Medicine, Uganda Martyrs Hospital Lubaga, P.O.BOX

14130, Kampala, Uganda

Full list of author information is available at the end of the article
} 


\section{Background}

\section{Burden of asthma and COPD: Globally and in sub-Saharan} Africa

The global burden of chronic respiratory disorders (CRD) particularly asthma and chronic obstructive pulmonary disease (COPD) continues to increase especially in low-and middle income countries (LMIC), posing a substantial public health threat [1]. The 2015 Global Burden of Diseases, Injuries and Risk Factors (GBD) study reported an increase in the prevalence of asthma and COPD by $12.6 \%$ and $44.2 \%$ respectively from 1990 to 2015. This was associated with an increased rate of mortality due to COPD [2]. In 2015, 3.2 million people and 0.4 million people died from COPD and asthma worldwide respectively. This represents an $11.6 \%$ increase in COPD-related deaths and a $26.7 \%$ decrease in asthmarelated deaths when compared to estimates in 1990 [2]. The majority of these deaths were reported in LMIC. Smoking (both active and passive), ambient particulate matter, household pollution and occupational triggers were identified as the key risk factors and contributors to DALYS for both asthma and COPD [2].

Increasing trends of morbidity due to asthma and COPD have also been reported in sub-Saharan Africa (SSA). Regarding the prevalence of asthma in Africa, a systematic review by Adeloye et al. [3] of 45 relevant studies published between 1990 and 2012 estimated about 74.4 million, 94.8 million and 119.3 million asthma cases in the total population in 1990, 2000 and 2010 respectively. A higher crude prevalence of asthma was noted in the urban areas compared to the rural areas.

A systematic review conducted in 2011 by Finney et al. [4] reported estimates of COPD to vary between 4 and 25\%. This data was obtained from 9 studies that were performed in 4 countries (Nigeria, South Africa, Malawi and Cape Verde). Only one study used a population based representative sampling approach. Another systematic review performed by Adeloye et al. [5] in 2012 that included 13 eligible studies (5 of which were based on spirometry data) reported a prevalence of COPD of $13.4 \%$ using spirometric data and $4 \%$ using non spirometric data. The most recent community surveys that have been conducted between 2013 to-date to ascertain the burden of COPD in Cameroon, Malawi, Nigeria, Uganda and Tanzania have reported prevalence of $2.4 \%, 7.7 \%, 4.2 \%, 16.2 \%$ and $17.5 \%$ respectively [6-10].

To effectively address the public health concern that asthma and COPD pose in SSA, health systems should be well structured to prevent, diagnose early and optimally manage these conditions. Consistent availability of affordable medicines and diagnostic tests is a fundamental component in the management of asthma and COPD in clinical practice. The Global Initiative for Asthma
(GINA) and Global Initiative for Chronic Obstructive Lung Disease (GOLD) guidelines recommend the use of inhaled short acting beta agonists (SABA), inhaled short acting anti muscarinic agents (SAMA), inhaled long acting anti muscarinic agents (LAMA), inhaled long acting beta agonists (LABA) and LAMA combinations, inhaled corticosteroid (ICS)-LABA combinations, ICS monotherapies, oral methylxanthines, oral leukotriene receptor antagonists (LTRA), pneumococcal vaccination and phosphodiesterase-4 inhibitors as the mainstay pharmacological therapies in the management of asthma and COPD $[11,12]$.

Citing the evident increase in morbidity and mortality due to asthma and COPD in SSA, it is essential to document the current status of availability and affordability of these medicines and diagnostic tests recommended for management of asthma and COPD in SSA. This will help in the guiding the formulation and implementation of pragmatic solutions to address the challenges of poor availability and high cost of these medicines and diagnostic tests.

Availability and affordability of medicines and diagnostic tests recommended for the management of asthma and COPD in SSA has not been systematically studied to-date. We, therefore undertook a systematic review of relevant original studies performed between 2000 and March 2018 that investigated availability and affordability of these medicines and diagnostic tests in SSA.

\section{Methods}

A comprehensive literature search of PubMed, Scopus and African Journal Online was performed for original research articles in English language performed between 2000 and March 2018 with an objective of determining the scope of availability and affordability of key medicines and diagnostic tests recommended in the management of asthma and COPD in SSA. References of the selected original research articles and published review articles were further searched for additional original research articles. We also searched the first 1000 Google scholar searches for original research articles.

Studies included were original research articles that contained information about availability and affordability of any known important medicine and diagnostic test for the management of asthma and COPD, was conducted in SSA between 2000 and March 2018 and published in English language.

Availability of any medicine (s) or diagnostic test (s) was expressed as a percentage of health facilities where it was present at the time of the study. Affordability was expressed as the total number of days' wages it would cost the lowest paid unskilled government worker to pay 
for a diagnostic test or a months' cost of the medicine. This information was documented precisely as reported in the eligible original research article.

The classes of medicines of interest were those belonging to these categories: inhaled SABA, SAMA, LAMA, ICS, SABA-SAMA combinations, LABALAMA combinations, ICS-LABA combinations, oral methylxanthines and LTRA. Studies that also contained information about the availability and affordability of spacer devices were also included due to their role in drug delivery especially in young children and elderly patients. The diagnostic tests of interest were spirometry and peak expiratory flow devices.

The following search terms were used: access OR affordability OR pricing OR cost OR availability AND "essential medicines" OR drugs OR therapies OR medicines AND tests OR diagnostic OR imaging AND asthma OR "chronic obstructive pulmonary disease" OR COPD OR "chronic respiratory disorders" AND Africa. The titles and abstracts of all studies were initially assessed for eligibility. Full texts of those studies that initially met the inclusion criteria were obtained and screened by 2 independent reviewers (DK and RES) and then exported to Endnote citation manager. Three extra authors (RN, WW and BK) independently reviewed the selected original research articles for the eligibility and key information about study setting and design and information about availability and affordability using a data extraction form. We excluded original research articles published in other languages other than English, studies whose full texts could not be accessed for full analysis and review articles.

The methodological quality of the identified studies was assessed by 2 independent reviewers (DK and RES) using the Newcastle-Ottawa scale. A maximum score of 6 and 9 was used for the selected cross sectional and case-control and cohort studies respectively [13].

The PRISMA guidelines for the reporting of systematic reviews were followed (Table 1) [14]. This systematic review was registered in PROSPERO (registration number: CRD42018093391).

\section{Results}

A total of 796 published articles were identified after searching the 3 databases. Thirty-two duplicates were removed, leaving 764 articles. The titles and abstracts of 764 articles were screened. Of these, 737 articles were excluded because they lacked the information of interest. Full texts of 27 articles were assessed for eligibility and only 6 articles met the inclusion criteria. Three papers were added after searching Google Scholar, references of the eligible original articles and published review articles, making a total of 9 eligible original research articles which were included in the systematic review [15-23] (Fig. 1-Flow diagram summarizing the identification of eligible articles).

\section{Study characteristics and methodological quality}

All eligible studies were cross sectional in nature. Six of the studies (67\%) were performed in a single African country (Malawi, Uganda-2 different studies, Nigeria, Ghana, South Africa) [15-17, 19, 20, 23]. The rest were multi-country studies performed in both an African country and other LMIC outside Africa [18, 21, 22]. In these multi-country studies, only data reported from the African countries was obtained.

Only 1 study was assigned the maximum score of 6 [16], with the rest scoring $5(\mathrm{n}=2,22.2 \%)[19,20]$ and $4(\mathrm{n}=6,66.7 \%)[15,17,18,21-23]$ on methodological quality.

The eligible original studies included in the systematic review are summarised in Table 2.

\section{Availability of essential medicines used in management of exacerbations of both asthma and COPD (inhaled SABA and SAMA monotherapy) Inhaled SABA monotherapies}

Six studies evaluated the availability of inhaled SABA (inhaled salbutamol) $[16,17,19-22]$ which ranged from $19.9 \%$ in one study performed in Uganda [20] to $100 \%$ in Eriteria [22]. Low rates of availability of inhaled SABA were reported in a multi-centre study of 8 SSA countries (14\% and $47 \%$ in public and private sector respectively) [21], Benin (33.3\%) [22] and Ghana (39.1\%) [19]. Comparable rates of moderate availability were reported in another study in Uganda (75\%) [16] and other studies performed in Sudan (71.4\%) [22] and Nigeria (76.5\%) [17].

\section{Inhaled SAMA monotherapy}

Availability of inhaled SAMA (ipratropium bromide) was documented to be very low, with levels of $0 \%$ in Benin and Eriteria [22], 2.9\% in Nigeria [17], 4.5\% in Ghana [19], 12.3\% in Uganda [16] and 14.3\% in Sudan [22].

\section{Availability of medicines used in symptom control of both asthma and COPD (ICS, ICS-LABA combinations, low dose theophylline, LTRA and inhaled tiotropium (LAMA) \\ Ics}

Availability of ICS reported by 7 studies [15-20, 22] ranged from $0 \%$ in a multicenter study of 7 SSA countries [18] and in another study performed in Malawi [15] to $45.5 \%$ in Uganda [16]. Very low availability of ICS was noted in another study performed in Uganda (1.5\%) [20], a multi-country study involving 3 SSA countries 
Table 1 Prisma checklist for the systematic review

\begin{tabular}{|c|c|c|}
\hline Section/topic & \# & Checklist item \\
\hline \multicolumn{3}{|l|}{ Title } \\
\hline Title & 1 & Identify the report as a systematic review, meta-analysis, or both \\
\hline \multicolumn{3}{|l|}{ Abstract } \\
\hline Structured summary & 2 & $\begin{array}{l}\text { Provide a structured summary including, as applicable: background; objectives; data } \\
\text { sources; study eligibility criteria, participants, and interventions; study appraisal and } \\
\text { synthesis methods; results; limitations; conclusions and implications of key findings; } \\
\text { systematic review registration number }\end{array}$ \\
\hline
\end{tabular}

Introduction

Rationale

3 Describe the rationale for the review in the context of what is already known

Objectives

4 Provide an explicit statement of questions being addressed with reference to participants, 5 interventions, comparisons, outcomes, and study design (PICOS)

Methods

Protocol and registration

Eligibility criteria

Information sources

5 Indicate if a review protocol exists, if and where it can be accessed (e.g., Web address), and, 7 if available, provide registration information including registration number

6 Specify study characteristics (e.g., PICOS, length of follow-up) and report characteristics (e.g., years considered, language, publication status) used as criteria for eligibility, giving rationale

7 Describe all information sources (e.g., databases with dates of coverage, contact with 5 study authors to identify additional studies) in the search and date last searched

Search

8 Present full electronic search strategy for at least one database, including any limits used, such that it could be repeated

Study selection

9 State the process for selecting studies (i.e., screening, eligibility, included in systematic review, and, if applicable, included in the meta-analysis)

Data collection process

10 Describe method of data extraction from reports (e.g., piloted forms, independently, in duplicate) and any processes for obtaining and confirming data from investigators

Data items

11 List and define all variables for which data were sought (e.g., PICOS, funding sources) and any assumptions and simplifications made

Risk of bias in individual studies

12 Describe methods used for assessing risk of bias of individual studies (including specification of whether this was done at the study or outcome level), and how this information is to be used in any data synthesis

Summary measures

13 State the principal summary measures (e.g., risk ratio, difference in means)

1 3

Synthesis of results

14 Describe the methods of handling data and combining results of studies, if done, including measures of consistency (e.g., $I^{2}$ ) for each meta-analysis

Risk of bias across studies

15 Specify any assessment of risk of bias that may affect the cumulative evidence (e.g., publication bias, selective reporting within studies)

Additional analyses

16 Describe methods of additional analyses (e.g., sensitivity or subgroup analyses, metaregression), if done, indicating which were pre-specified

Results

Study selection

17 Give numbers of studies screened, assessed for eligibility, and included in the review, with reasons for exclusions at each stage, ideally with a flow diagram

Study characteristics

18 For each study, present characteristics for which data were extracted (e.g., study size, PICOS, follow-up period) and provide the citations

Risk of bias within studies

19 Present data on risk of bias of each study and, if available, any outcome level assessment (see item 12)

Results of individual studies

20 For all outcomes considered (benefits or harms), present, for each study: (a) simple summary data for each intervention group (b) effect estimates and confidence intervals, ideally with a forest plot

Synthesis of results

21 Present results of each meta-analysis done, including confidence intervals and measures of consistency

Risk of bias across studies

22 Present results of any assessment of risk of bias across studies (see item 15)

Additional analysis

23 Give results of additional analyses, if done (e.g., sensitivity or subgroup analyses, metaregression [see item 16])

Discussion

Summary of evidence

24 Summarize the main findings including the strength of evidence for each main outcome; 11-13 consider their relevance to key groups (e.g., healthcare providers, users, and policy makers) 
Table 1 (continued)

\begin{tabular}{lllc}
\hline Section/topic & $\#$ & Checklist item & Reported on page \# \\
\hline Limitations & $25 \begin{array}{c}\text { Discuss limitations at study and outcome level (e.g., risk of bias), and at review-level (e.g., } \\
\text { incomplete retrieval of identified research, reporting bias) }\end{array}$ & 13 \\
Conclusions & $26 \begin{array}{c}\text { Provide a general interpretation of the results in the context of other evidence, and } \\
\text { implications for future research }\end{array}$ & 14 \\
$\begin{array}{l}\text { Funding } \\
\text { Funding }\end{array}$ & $27 \begin{array}{c}\text { Describe sources of funding for the systematic review and other support (e.g., supply of } \\
\text { data); role of funders for the systematic review }\end{array}$ & 15 \\
\hline
\end{tabular}

From: Moher D, Liberati A, Tetzlaff J, Altman DG, The PRISMA Group (2009). Preferred Reporting Items for Systematic Reviews and Meta-Analyses: The PRISMA Statement. PLoS Med 6(7): e1000097

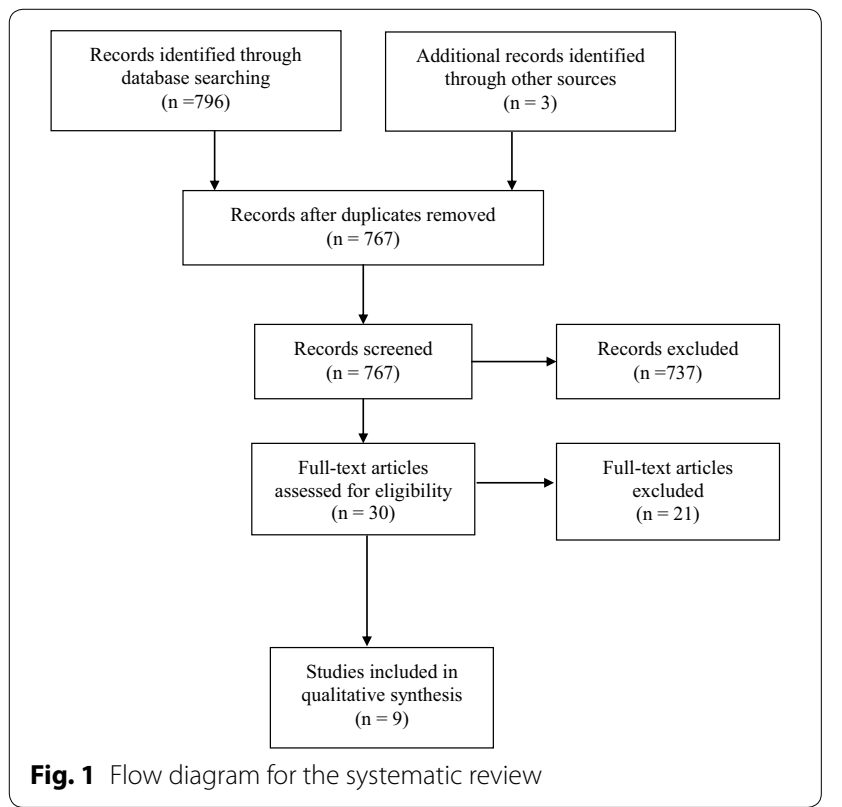

(Benin-16.7\%, Sudan-21.4\% and Eriteria-33.3\%) [22], Ghana (17.4\%) [19], 23.5\% (Nigeria) [17] and the private sector in Malawi (38\%) [15].

\section{Inhaled ICS-LABA combinations}

Only 2 studies performed in Uganda [16] and Nigeria [17] assessed availability of ICS-LABA combinations; documenting comparable low rates of $46.9 \%$ and $50 \%$ respectively.

\section{Oral low dose theophylline and LTRA}

Varying levels of availability of oral theophylline and LTRA were reported by 2 studies [16, 17]. In a study performed in Uganda, availability of oral theophylline and LTRA was $16.9 \%$ and $60.8 \%$ respectively [16] compared to $76.5 \%$ and $5.9 \%$ respectively in a study performed in Nigeria [17].

\section{Inhaled LAMA (tiotropium)}

Only 1 study investigated availability of inhaled tiotropium, reporting its absence in all health facilities surveyed [16].

\section{Availability of medicines used in symptom control of COPD only (inhaled LABA monotherapy, SABA-SAMA combinations and LAMA and LABA combinations)} Availability of all the above medicines was studied by only 1 study, documenting comparable very low levels of availability of inhaled LABA monotherapies and SABA-SAMA combinations of $10 \%$ and $10.8 \%$ respectively. No surveyed health facility had any inhaled LABA-LAMA combination [16].

No study investigated the availability of LABALAMA-ICS triple combinations, pneumococcal vaccines and phosphodiesterase 4 inhibitors which are essential medicines in the management of COPD.

\section{Spacers}

Due to the important role of spacers in drug delivery in the management of asthma and COPD in children and elderly patients, we also included information about their availability from 4 studies [16, 17, 19, 23]. With the exception of a study performed in South Africa where spacers were available in $72.9 \%$ of the surveyed 46 primary healthcare facilities, the rest of the studies reported very low levels of availability of $0 \%$ in Ghana [19], $18.5 \%$ and $19.2 \%$ for adult and paediatric spacers respectively in Uganda [16] and 20.6\% in Nigeria [17].

\section{Availability of diagnostic tests for asthma and COPD}

Only 4 studies contained findings about the availability of the diagnostic tests $[16,17,19,23]$. Availability of peak expiratory flow devices was reported about in all the 4 studies $(6.7 \%$ in Uganda, 13\% in Ghana, 38\% in Nigeria and $53.6 \%$ in South Africa). Availability of 


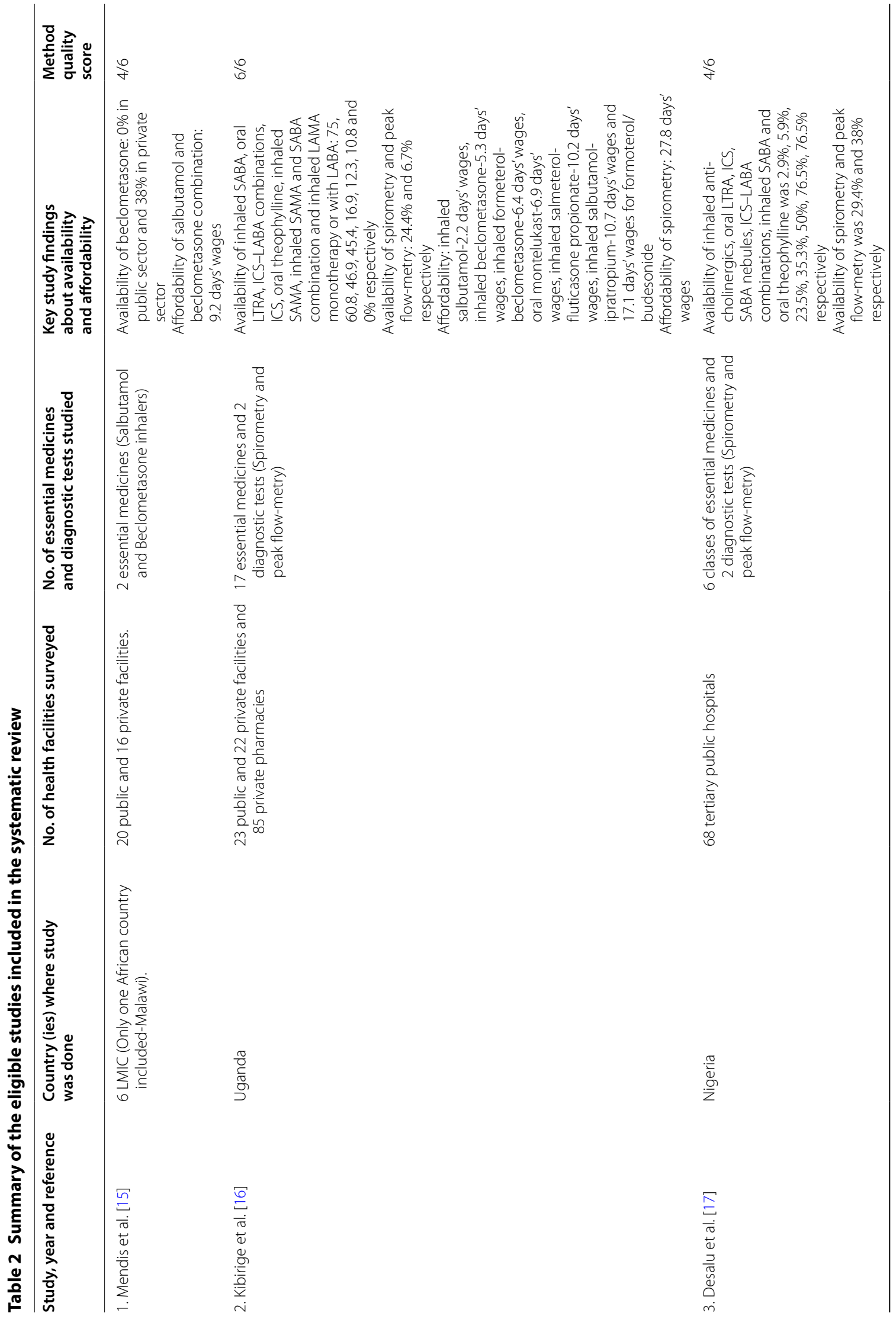




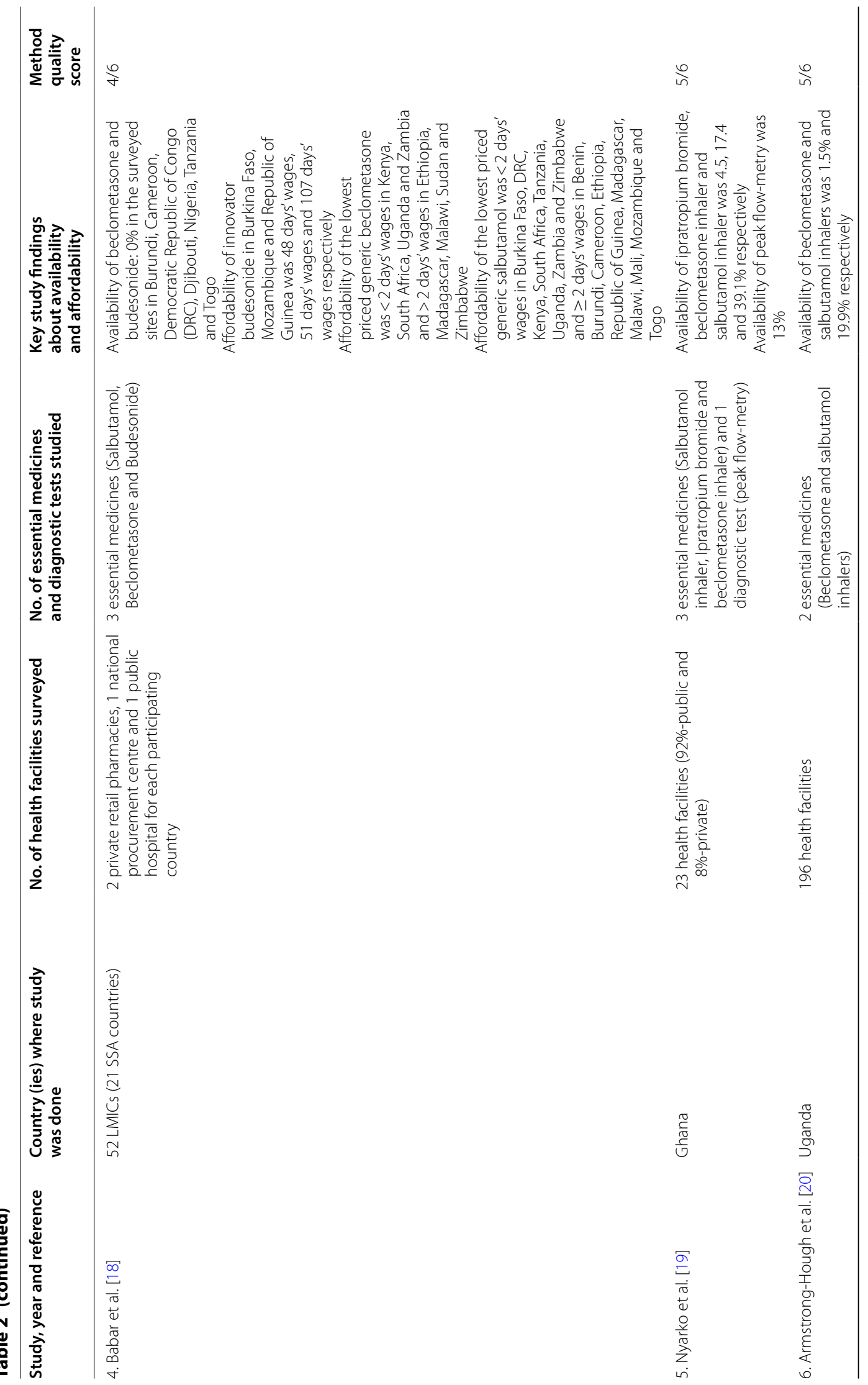




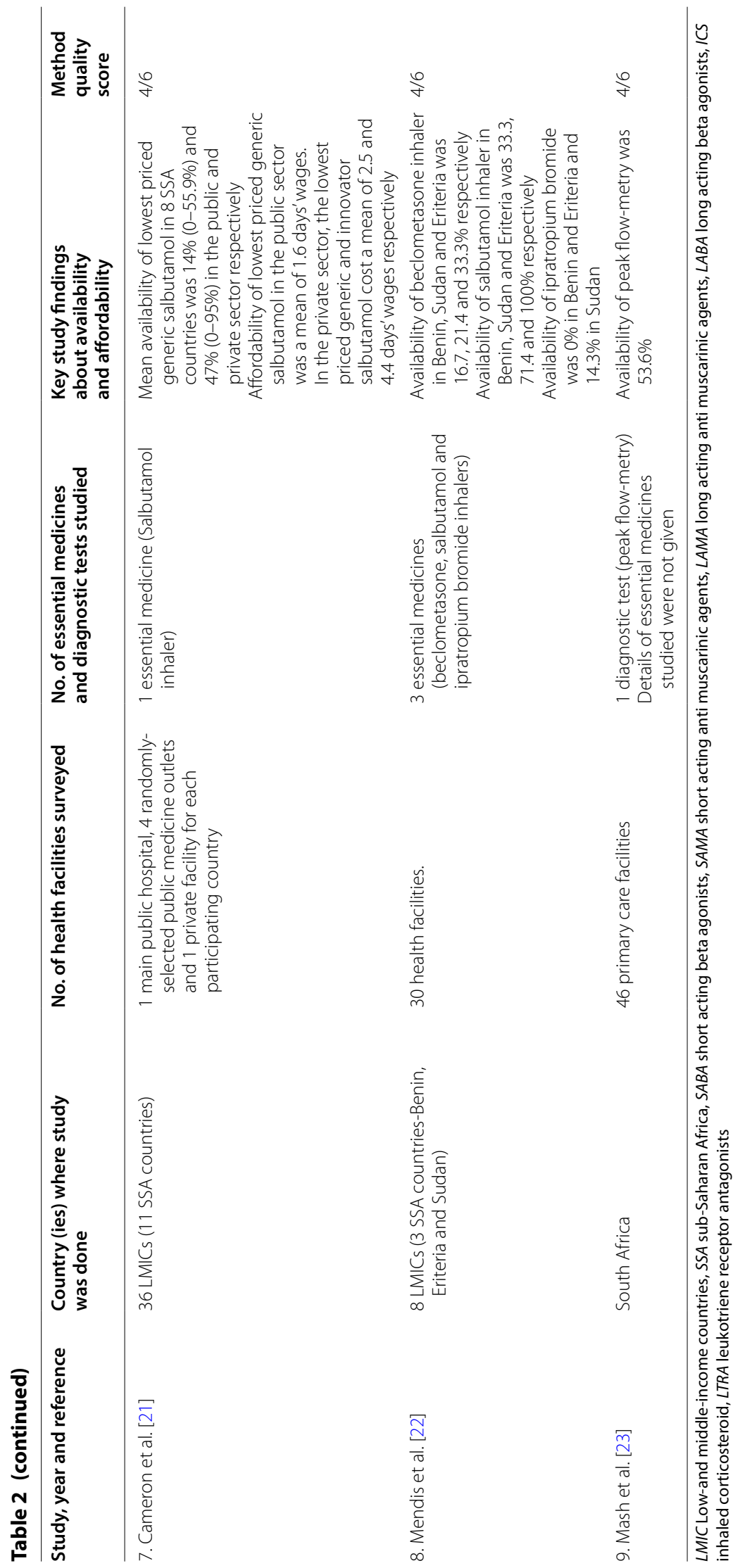


spirometry was reported only by studies performed in Uganda (24.4\%) [16] and Nigeria (29.4\%) [17].

\section{Affordability of medicines used in management of exacerbations of both asthma and COPD (inhaled SABA and SAMA monotherapies) \\ Inhaled SABA (salbutamol)}

In a multi-centre study by Babar et al. [18] that included 22 countries in SSA, the lowest priced generic (LPG) inhaled salbutamol cost $<2$ days' wages in 8 countries (Burkina Faso, Democratic Republic of Congo, Kenya, South Africa, Tanzania, Uganda, Zambia and Zimbabwe) and $\geq 2$ days' wages in 10 countries (Benin, Burundi, Cameroon, Ethiopia, Republic of Guinea, Madagascar, Malawi, Mali, Mozambique and Togo). Another multicentre study that included information from 8 countries in SSA reported the lowest priced generic (LPG) salbutamol inhaler to cost a mean of 1.6 days' wages and 2.5 days' wages in the public and private sector respectively. The innovator brand of salbutamol inhaler cost a mean of 4.4 days' wages [21]. In another study done in Uganda, the LPG salbutamol inhaler cost 2.2 days' wages [16].

\section{Inhaled SAMA monotherapy (ipratropium)}

The LPG SAMA (inhaled ipratropium) as reported by only 1 study cost 13.7 and 10.7 days' wages for the $20 \mu \mathrm{g}$ and $40 \mu \mathrm{g}$ respectively [16].

\section{Affordability of medicines used in symptom control of both asthma and COPD (ICS, ICS-LABA combinations, low dose theophylline, LTRA and inhaled LAMA) \\ Ics}

A disparity in the affordability of generic and innovator ICS was observed in the study by Babar et al. [18] that included information from 21 countries in SSA. The LPG beclometasone cost $<2$ days' wages in Kenya, South Africa, Uganda and Zambia and $\geq 2$ days' wages in Ethiopia, Madagascar, Malawi, Sudan and Zimbabwe. The cost of innovator budesonide was 48 days' wages, 51 days' wages and 107 days' wages in Burkina Faso, Mozambique and Republic of Guinea respectively. In Uganda, the LPG inhaled beclometasone, fluticasone propionate and budesonide cost 5.3 days' wages and 8 days' wages respectively [16].

\section{ICS-LABA combinations, SAMA-SABA combinations, Iow dose theophylline and LTRA}

Only 1 study contained information about the affordability of the above medicines [16]. Regarding ICS-LABA combinations, the reported cost of the LPG formoterol/beclometasone, salmeterol/fluticasone propionate and formoterol-budesonide was 6.4 days' wages, 10.2 days' wages and 17.1 days' wages respectively. Inhaled SAMA-SABA (ipratropium-salbutamol combination) cost 10.7 days' wages while oral LTRA and low dose theophylline both cost 6.9 days' wages. Adult and paediatric spacers cost 12.9 and 7.5 days' wages respectively [16].

\section{Affordability of diagnostic tests of asthma and COPD}

One study evaluated affordability of spirometry which cost up to 27.8 days' wages [16].

\section{Discussion}

To our knowledge, this systematic review provides the first comprehensive assessment of availability and affordability of internationally recommended medicines and diagnostic tests in management of asthma and COPD in SSA. It also evidently demonstrates that studies investigating this key area in SSA are still limited and availability of affordable medicines and diagnostic tests still remains a substantial challenge in clinical practice in the region.

We noted considerable heterogeneity in the study results and health facilities surveyed. Poor availability was widely noted with SAMA, SAMA-SABA combinations, ICS, LAMA, LAMA-LABA combinations and diagnostic tests (spirometry and peak expiratory flow devices). The paucity of studies investigating access to LAMA and LAMA-LABA combinations, which are vital medicines in reducing COPD symptoms and future exacerbations is also revealing. This poor access to medicines for asthma and COPD has been highlighted in SSA and other LMIC and it carries substantial public health implications [24-27]. Availability of SABA (salbutamol inhaler) and LABA-ICS combination was $>70 \%$ in Uganda, Nigeria, Sudan and Eriteria $[16,17,22]$ and $>45 \%$ in Uganda and Nigeria [16, 17]. The fairly good availability could explain the frequent use of SABA that has been described in patients with asthma and COPD in clinical practice.

Poor access to spirometry as a cornerstone for diagnosis and assessment of treatment response in patients with asthma and COPD as noted by this systematic review still remains a challenge. The poor availability of spirometry in health facilities and inadequate proficiency in interpretation of spirometric readings by healthcare workers in clinical practice has been widely documented as an impediment to optimal management of asthma and COPD in SSA [26-28]. The absence of adequate numbers of skilled personnel to perform spirometry and interpret its findings could also partly explain its low availability in SSA.

A dearth of studies about affordability of the medicines and diagnostic tests for asthma and COPD in SSA is also of great concern. The majority of the medicines 
(controller therapies) especially in the private sector and innovator brands remain unaffordable for most patients in SSA.

Several reasons could explain the poor availability and high costs of the majority of the medicines and diagnostic tests in SSA. There exists a knowledgepractice gap among healthcare practitioners in SSA resulting into patterns of clinical practice that are not in conformity with international treatment guidelines $[27,29]$. Low prescribing rates of these medicines by healthcare practitioners directly or indirectly influence their availability both in the public and private sector. Exclusion of these medicines from the national essential medicine lists (EML) and treatment guidelines could also explain the poor availability in SSA. One survey that investigated the number of asthma and COPD essential medicines on the national EML of 32 LMIC (including some countries in SSA) noted that the median number of essential medicines was 7 , with a range of 0-22. Notably, no African country included LABA to their national EML. Few low income countries (LIC) included inhaled anti cholinergic agents (20\%). None of the LIC included therapies recommended in step 4 and 5 of asthma management by the GINA guidelines. Only a third of them included at least 1 medicine recommended in the step 2 and 3 of COPD management by the GOLD guidelines [30].

The high cost of the medicines and diagnostic tests in SSA could be explained by lack of local price regulatory frameworks or legislation, absence or poor access to national health insurance schemes that can provide the medicines at subsided fees, low scale of local pharmaceutical production of generic medicines and absence of public and private co-financing initiatives to reduce costs of drugs.

\section{Strengths and limitations of the systematic review}

One significant strength of this systematic review is being the first review to offer comprehensive information about the extent of availability and affordability of medicines and diagnostic tests of asthma and COPD in SSA. Despite this, some of the limitations are heterogeneity of the study findings and health facilities surveyed and the low methodological quality of the eligible original studies.

\section{Conclusion}

A better understanding of the magnitude and reasons to explain the challenge of poor availability and high cost of these medicines and diagnostic tests in SSA is important to guide better implementation of pragmatic solutions and guidelines. Poor availability and unaffordability of medicines and diagnostic tests recommended for the management of asthma and
COPD in SSA could be addressed through increasing awareness about the burden of both conditions and their optimal management among healthcare practitioners, improving local manufacturing of cheap good quality generic medicines, updating national EML and treatment guidelines, improving supply chain and forecast and sustained and equitable government financing of health budgets. Implementation of health policies like national health insurance schemes, regulation of local retail prices of chronic diseases and introduction of preferential registration procedures for locally manufactured generic drugs by drug regulatory institutions can help address the challenge of high costs of medicines of asthma and COPD in SSA.

Due to differences in economic status of countries in SSA, more robust country-specific large studies about access to affordable essential medicines and diagnostic tests are needed to further appreciate the magnitude of this public health problem in this region.

\begin{abstract}
Abbreviations
CRD: chronic respiratory disorders; COPD: chronic obstructive pulmonary disease; LMIC: low-and middle income countries; GBD: Global Burden of Diseases; SSA: sub-Saharan Africa; GINA: Global Initiative for Asthma; GOLD: Global Initiative for Chronic Obstructive Lung Disease; SABA: short acting beta agonists; SAMA: short acting anti muscarinic agents; LAMA: long acting anti muscarinic agents; LABA: long acting beta agonists; ICS: inhaled corticosteroid; LTRA: oral leukotriene receptor antagonists.
\end{abstract}

\section{Authors' contributions \\ DK-conceived the idea and wrote the initial manuscript, DK and RES- performed initial screening of the online searches for eligible research articles, RN, WW and BK-independently reviewed selected articles for eligibility and key information, RES, RN, WW and BK-reviewed the initial manuscript. All authors read and approved the final manuscript.}

\section{Author details}

${ }^{1}$ Department of Medicine, Uganda Martyrs Hospital Lubaga, P.O.BOX 14130, Kampala, Uganda. ${ }^{2}$ Non-communicable Diseases Theme, Medical Research Council/Uganda Virus Research Institute and London School of Hygiene and Tropical Medicine Uganda Research Unit, Entebbe, Uganda. ${ }^{3}$ Makerere University Lung Institute, Makerere University College of Health Sciences, Kampala, Uganda. ${ }^{4}$ Department of Paediatrics and Child Health, Makerere University College of Health Sciences, Kampala, Uganda. ${ }^{5}$ Division of Pulmonology, Department of Medicine, Mulago National Referral and Teaching Hospital, Kampala, Uganda.

\section{Acknowledgements \\ None.}

Competing interests

The authors declare that they have no competing interests.

\section{Availability of data and materials}

The studies included in this systematic review are available from the corresponding author on reasonable request and also available online.

\section{Consent for publication}

Not applicable.

Ethics approval and consent to participate

Ethical approval was not required since this is a systematic review of published studies. 


\section{Funding}

No funding was received for this systematic review.

\section{Publisher's Note}

Springer Nature remains neutral with regard to jurisdictional claims in published maps and institutional affiliations.

Received: 28 August 2018 Accepted: 20 February 2019

Published online: 07 March 2019

\section{References}

1. WHO. Chronic respiratory disorders. 2018. http://www.whoint/respirator y/en/. Accessed 2 Apr 2018.

2. Soriano JB, Abajobir AA, Abate KH, Abera SF, Agrawal A, Ahmed MB, Aichour AN, Aichour I, Aichour MTE, Alam K, et al. Global, regional, and national deaths, prevalence, disability-adjusted life years, and years lived with disability for chronic obstructive pulmonary disease and asthma, 1990-2015: a systematic analysis for the Global Burden of Disease Study 2015. Lancet Respir Med. 2017;5:691-706.

3. Adeloye D, Chan KY, Rudan I, Campbell H. An estimate of asthma prevalence in Africa: a systematic analysis. Croat Med J. 2013;54:519-31.

4. Finney LJ, Feary JR, Leonardi-Bee J, Gordon SB, Mortimer K. Chronic obstructive pulmonary disease in sub-Saharan Africa: a systematic review. Int J Tuberc Lung Dis. 2013;17:583-9.

5. Adeloye D, Basquill C, Papana A, Chan KY, Rudan I, Campbell H. An estimate of the prevalence of COPD in Africa: a systematic analysis. J Chron Obstruct Pulmon Dis. 2015;12:71-81.

6. Pefura-Yone EW, Kengne AP, Balkissou AD, Magne-Fotso CG, Ngo-Yonga M, Boulleys-Nana JR, Efe-de-Melingui NR, Ndjeutcheu-Moualeu PI, Mbele-Onana CL, Kenmegne-Noumsi EC, et al. Prevalence of obstructive lung disease in an African country using definitions from different international guidelines: a community based cross-sectional survey. BMC Res Notes. 2016:9:124.

7. Meghji J, Nadeau G, Davis KJ, Wang D, Nyirenda MJ, Gordon SB, Mortimer K. Noncommunicable lung disease in sub-Saharan Africa. A communitybased cross-sectional study of adults in Urban Malawi. Am J Respir Crit Care Med. 2016;194:67-76.

8. Obaseki DO, Erhabor GE, Gnatiuc L, Adewole OO, Buist SA, Burney PG. Chronic airflow obstruction in a Black African population: results of BOLD study, Ile-Ife, Nigeria. Copd. 2016;13:42-9.

9. van Gemert F, Kirenga B, Chavannes N, Kamya M, Luzige S, Musinguzi P, Turyagaruka J, Jones R, Tsiligianni I, Williams S, et al. Prevalence of chronic obstructive pulmonary disease and associated risk factors in Uganda (FRESH AIR Uganda): a prospective cross-sectional observational study. Lancet Glob Health. 2015;3:e44-51.

10. Magitta NF, Walker RW, Apte KK, Shimwela MD, Mwaiselage JD, Sanga AA, Namdeo AK, Madas SJ, Salvi SS. Prevalence, risk factors and clinical correlates of COPD in a rural setting in Tanzania. Eur Respir J. 2018:51:1700182.

11. GINA. 2018 GINA report, global strategy for asthma management and prevention; 2018. https://ginasthma.org/wp-content/uploads/2018/04/ wms-GINA-2018-report-V1.3-002.pdf. Accessed 27 Feb 2019.

12. GOLD. Global strategy for diagnosis, management and prevention of chronic obstructive pulmonary disease (2018 report); 2018. https://goldc opd.org/wp-content/uploads/2017/11/GOLD-2018-v6.0-FINAL-revis ed-20-Nov_WMS.pdf. Accessed 27 Feb 2019.

13. Wells G, Shea B, O'Connell D, Peterson J, Welch V, Losos M, Tugwell P. The Newcastle-Ottawa Scale (NOS) for assessing the quality of nonrandomised studies in meta-analyses. http://www.ohrica/programs/ clinical_epidemiology/oxfordasp. Accessed 7 July 2018.
14. Moher D, Shamseer L, Clarke M, Ghersi D, Liberati A, Petticrew M, Shekelle $P$, Stewart LA. Preferred reporting items for systematic review and metaanalysis protocols (PRISMA-P) 2015 statement. Syst Rev. 2015;4:1.

15. Mendis S, Fukino K, Cameron A, Laing R, Filipe A Jr, Khatib O, Leowski J, Ewen $M$. The availability and affordability of selected essential medicines for chronic diseases in six low- and middle-income countries. Bull World Health Organ. 2007:85:279-88.

16. Kibirige D, Kampiire L, Atuhe D, Mwebaze R, Katagira W, Muttamba W, Nantanda R, Worodria W, Kirenga B. Access to affordable medicines and diagnostic tests for asthma and COPD in sub Saharan Africa: the Ugandan perspective. BMC Pulm Med. 2017;17:179.

17. Desalu OO, Onyedum CC, Iseh KR, Salawu FK, Salami AK. Asthma in Nigeria: are the facilities and resources available to support internationally endorsed standards of care? Health Policy. 2011;99:250-4.

18. Babar ZU, Lessing C, Mace C, Bissell K. The availability, pricing and affordability of three essential asthma medicines in 52 low- and middleincome countries. Pharmacoeconomics. 2013;31:1063-82.

19. Nyarko KM, Ameme DK, Ocansey D, Commeh E, Markwei MT, Ohene SA. Capacity assessment of selected health care facilities for the pilot implementation of Package for Essential Non-communicable Diseases (PEN) intervention in Ghana. Pan Afr Med J. 2016;25:16.

20. Armstrong-Hough M, Kishore SP, Byakika S, Mutungi G, Nunez-Smith M, Schwartz JI. Disparities in availability of essential medicines to treat noncommunicable diseases in Uganda: a Poisson analysis using the Service Availability and Readiness Assessment. PLoS ONE. 2018;13:e0192332.

21. Cameron A, Ewen M, Ross-Degnan D, Ball D, Laing R. Medicine prices, availability, and affordability in 36 developing and middle-income countries: a secondary analysis. Lancet. 2009;373:240-9.

22. Mendis S, Al Bashir I, Dissanayake L, Varghese C, Fadhil I, Marhe E, Sambo B, Mehta F, Elsayad H, Sow I, et al. Gaps in capacity in primary care in low-resource settings for implementation of essential noncommunicable disease interventions. Int J Hypertens. 2012;2012:584041.

23. Mash B, Rhode H, Pather M, Ainslie G, Irusen E, Bheekie A, Mayers P. Quality of asthma care: western Cape province, South Africa. S Afr Med J. 2009:99:892-6.

24. Bissell K, Perrin C, Beran D. Access to essential medicines to treat chronic respiratory disease in low-income countries. Int J Tuberc Lung Dis. 2016;20:717-28.

25. Beran D, Zar HJ, Perrin C, Menezes AM, Burney P. Burden of asthma and chronic obstructive pulmonary disease and access to essential medicines in low-income and middle-income countries. Lancet Respir Med. 2015:3:159-70

26. van Gemert FA, Kirenga BJ, Gebremariam TH, Nyale G, de Jong C, van der Molen T. The complications of treating chronic obstructive pulmonary disease in low income countries of sub-Saharan Africa. Expert Rev Respir Med. 2018:12:227-37

27. Onyedum C, Ukwaja K, Desalu O, Ezeudo C. Challenges in the management of bronchial asthma among adults in Nigeria: a systematic review. Ann Med Health Sci Res. 2013;3:324-9.

28. Mehrotra A, Oluwole AM, Gordon SB. The burden of COPD in Africa: a literature review and prospective survey of the availability of spirometry for COPD diagnosis in Africa. Trop Med Int Health. 2009;14:840-8.

29. Kirenga J, Okot-Nwang M. The proportion of asthma and patterns of asthma medications prescriptions among adult patients in the chest, accident and emergency units of a tertiary health care facility in Uganda. Afr Health Sci. 2012;12:48-53.

30. Bazargani YT, de Boer A, Leufkens HG, Mantel-Teeuwisse AK. Essential medicines for COPD and asthma in low and middle-income countries. Thorax. 2014;69:1149-51. 